\title{
NUMERICAL SIMULATION OF RAM EXTRUSION IN SHORT-FIBER-REINFORCED FRESH CEMENTITIOUS COMPOSITES
}

\author{
XiAngMing Zhou AND ZongJin Li
}

\begin{abstract}
A series of ram extrusion tests was carried out on a short-fiber-reinforced, semisolid, fresh cementitious composite. An elastoviscoplastic constitutive model is proposed for the extrudable fresh cementitious composite. It features the associative flow rule, a nonlinear strain rate-hardening law, and the von Mises yield criterion. The model is then implemented in ANSYS/LS-DYNA explicit finite element code.

Various ram extrusion processes of the fresh cementitious composite were simulated. It has been found that the extrusion load versus imposed displacement predictions agree well with the experimental results. The fresh paste flow, through the die entry and the die-land, is then interpreted in light of the evolution of the deformation and distribution of state variables, mainly based on numerical results and the ram extrusion mechanism.

The effects of extrusion ratio and extrusion velocity on extrusion load are also investigated, based on the mechanical properties of the fresh cementitious composite. The study indicates that the numerical procedure established, together with the constitutive model proposed, is applicable for describing ram extrusion of short-fiber-reinforced fresh cementitious composites, which might provide a numerical rheometric tool from which ram extrusion of elastoviscoplastic paste-like materials can be examined and quantified.
\end{abstract}

\section{Introduction}

Extrusion is a material processing technology for manufacturing semisolid paste-like products used in the mechanical, chemical, ceramic, food, and pharmaceutical industries. In the last two decades, it has been applied in the concrete industry as an economical, efficient, and environmentally friendly manufacturing method for short-fiber-reinforced cement-based construction materials and products [Shao et al. 1995; Shao and Shah 1997; Aldea et al. 1998; Li and Mu 1998; Li et al. 1999; 2001; 2004; Peled and Shah 2003]. Compared with the traditional concrete casting method, extrusion techniques can significantly improve the mechanical properties of the final products through the high shear and high compression processing environment within an extruder [Shao et al. 1995; Peled and Shah 2003].

There are two main extrusion methods: the continuous screw-driven method (screw extrusion) and the intermittent piston-driven method (ram extrusion). The screw-driven method allows continuous production. However, it is not generally suitable for small batch runs because of difficulties in cleaning the extruder [Aydin et al. 2000]. It may not even be possible for some materials, such as metal alloys, due to limitations in rheology. On the other hand, ram extrusion is favored for short runs and accurate

Keywords: elastoviscoplastic, constitutive model, rheology, strain rate, fiber-reinforced, fresh cementitious composite, ram extrusion, overstress, rate-dependent, flow stress, LS-DYNA.

The financial support from the Hong Kong Research Grant Council under grant 616008 and from the China Ministry of Science and Technology under grant 2009CB623200 is gratefully acknowledged. 


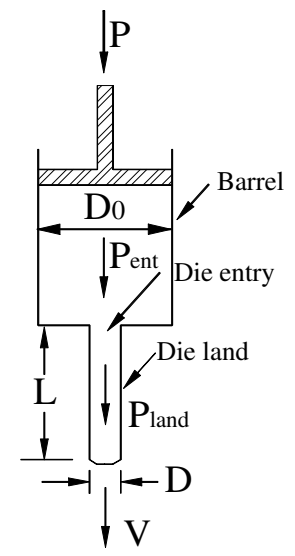

Figure 1. Schematic diagram of Benbow-Bridgwater ram extrusion mechanism.

dimensional control [Aydin et al. 2000]. In this method, a piston drives the highly viscous, semisolid fresh paste to flow through a die entry and then a die-land, thereby reducing the cross-sectional area of the paste and shaping it as desired.

Axisymmetric ram extrusion with a circular die entry, as shown in Figure 1, is a simple but effective method for tailoring mix proportions for an extrudable fiber-reinforced cementitious composite [Srinivasan et al. 1999; Zhou and Li 2005a]. The most widely used model for analyzing this type of ram extrusion data is based on a relationship known as the Benbow-Bridgwater equation [1993]:

$$
P=P_{\mathrm{ent}}+P_{\mathrm{land}}=2 \ln \left(\frac{D_{0}}{D}\right)\left(\sigma_{0}^{\prime}+\alpha V_{p}^{j}\right)+\frac{4 L}{D}\left(\tau_{0}^{\prime}+\beta V_{p}^{q}\right),
$$

where, as shown in Figure 1, $P$ is the overall pressure drop; the first term on the right in (1-1) is the die entry pressure drop, $P_{\text {ent }}$, representing that a plastic flow dominates at the die entry; the second term, $P_{\text {land }}$, is the die-land pressure drop, representing a slip flow in the die-land; $D_{0}$ is the barrel diameter; $D$ is the die-land diameter; $L$ is the die-land length; $V_{p}$ is the mean paste flow velocity in the die-land; $\sigma_{0}^{\prime}$ is the initial die entry yield stress when the paste velocity approaches zero; $\alpha$ is the die entry yield stress velocity factor; $j$ is an exponent that accounts for the nonlinear velocity dependence of the plastic flow in the die entry; $\tau_{0}^{\prime}$ is the initial die-land wall shear stress as the paste velocity approaches zero; $\beta$ is the die-land shear stress velocity factor, which accounts for the increase of the die-land wall shear stress with increasing paste flow velocity; and $q$ is an exponent that accounts for the nonlinear velocity dependence of the shear flow in the die-land. Note that $\sigma_{0}^{\prime}, \alpha, j, \tau_{0}^{\prime}, \beta$, and $q$ are not necessarily material parameters of the paste itself.

Though the Benbow-Bridgwater equation provides reasonably satisfactory ram extrusion predictions - mainly the relationship between the overall extrusion pressure drop and the mean paste flow velocity for many paste-like materials, such as ceramics and clays [Benbow et al. 1991; Benbow and Bridgwater 1993; Blackburn and Böhm 1994; Chou et al. 2003], it is basically a phenomenological model rather than a constitutive model for the ram extrusion process. It can only give an overall description of paste flow in a ram extruder with simple symmetrical geometry, based on the assumption of simple rigid plastic or rigid-viscoplastic material behavior for the paste. The ram extrusion process of paste-like materials with 
elastoviscoplastic constitutive behavior, in a nonaxisymmetric geometry, is difficult to directly describe with the Benbow-Bridgwater model. In this case, only numerical methods can give a full description of the ram extrusion process of materials exhibiting complicated elastoviscoplastic behavior.

In this study, a ram extrusion process using a short-fiber-reinforced, semisolid, fresh cementitious composite, with a circular die entry and die-land, is investigated through experimental as well as numerical analysis. The extrusion load versus the imposed ram displacement data, the evolution of the deformation, and the distribution of state variables within the paste flow are obtained through numerical simulation based on the ANSYS/LS-DYNA explicit finite element code, combined with an elastoviscoplastic constitutive model for the highly concentrated, short-fiber-reinforced fresh cementitious composite. Explicit analysis has been regarded as a more efficient solution than implicit analysis for large-deformation problems, such as material forming processes like extrusion [Antúnez 2000]. The numerical procedure and the constitutive model are mainly verified quantitatively by comparing numerical and experimental results with the extrusion load versus imposed ram displacement data. Then, the evolution of the deformation and state variables within the composite during the ram extrusion process is interpreted based on the numerical results and the ram extrusion mechanism. The effects of the extrusion ratio, the ratio between the area of the barrel and that of the die-land, and the extrusion velocity, the ram driving velocity, on the extrusion load are investigated based on the experimental and numerical results. The aim of this research is to test the applicability of the numerical procedure, combined with the elastoviscoplastic constitutive model, for describing the ram extrusion process of a short-fiber-reinforced, semisolid, fresh cementitious composite. The verified numerical procedure might also be used for modeling the forming processes of other paste-like materials, exhibiting elastoviscoplastic behavior, as well as for design and optimization of materials processing equipment.

\section{Experimental}

The experimental program within the scope of this study is essentially divided into two parts. The first part involves rheological measurements to establish an appropriate constitutive model for the extrudable fresh cementitious composite, through orifice and capillary extrusion [Zhou 2004; Zhou and Li 2005b] and upsetting tests [Zhou and Li 2006]. The second part involves ram extrusion tests of the fresh cementitious composite under various extrusion conditions, that is, extrusion ratios and/or extrusion velocities, that may appear in practice, from which the applied extrusion load with respect to the imposed ram displacement data are obtained. Both parts of the experimental program are carried out on the short-fiberreinforced fresh cementitious composite with mix formulation shown in Table 1. The basic constitutive compositions include ordinary Portland cement and slag with a weight ratio of $1: 1$ as the cementitious

\begin{tabular}{|cccccccc|}
\hline Cement & Slag & SS1 & SS2 & PVA & Methocel & ADVA & W/B \\
0.5 & 0.5 & 0.2 & 0.125 & $2 \%$ & $1 \%$ & $0.375 \%$ & 0.27 \\
\hline
\end{tabular}

Table 1. Mix formulation for the short-fiber-reinforced fresh cementitious composite used for extrusion (see text at the top of the next page for abbreviations). SS1, SS2, Methocel, and ADVA are presented in the weight ratio of the binder. PVA fiber is presented in the volume ratio of the paste. 
binder; $6 \mathrm{~mm}$ long polyvinyl alcohol (PVA) fiber with an average diameter of $14 \mu \mathrm{m}$; two types of silica sands (denoted SS1 and SS2, with a nominal diameter of 300-600 $\mu \mathrm{m}$ and $90-150 \mu \mathrm{m}$, respectively) from David Ball Comp. Ltd with a weight ratio of 8:5 as aggregates; Methocel powder, produced by Dow Chemical Comp. Ltd, as a rheology enhancer; and ADVA solution, made by W. R. Grace (HK) Ltd, as a superplasticizer. The water-to-binder weight ratio is 0.27 while the silica sands-to-binder weight ratio is 0.325 . The dosage of ADVA solid powder is $0.375 \%$ of the weight of the binder and it is incorporated into the composite in the form of an aqueous solution with a concentration of $30 \%$ by weight as supplied by the manufacturer. The total volume of PVA fibers incorporated is $2 \%$ of that of the readily mixed composite.

The mix formulation shown in Table 1 is a typical recipe for an extrudable shore fiber-reinforced cementitious composite [Srinivasan et al. 1999; Zhou and Li 2005a; 2005b]. To prepare the fresh cementitious composite for extrusion, cementitious binders (cement and slag), fibers, and Methocel powder are first mixed for 3 minutes in a dry state at a low speed by a Hobart planetary mixer. Then water, with ADVA superplasticizer solution, is added into the mixture and mixed for another 3 minutes. Once the dry powders are moistened, a higher speed is adjusted to for 3 minutes high shear mixing till a dough-like paste is produced. This dough-like paste is then used for various experiments, including orifice extrusion, capillary extrusion, ram extrusion, and upsetting tests as referred to in this study.

Note that short-fiber-reinforced fresh cementitious composites for extrusion purposes are largely different from traditional cement paste, mortar, suspension, slurry, fresh self-compacting concrete, and other fresh concretes which normally have larger water-to-binder ratios and better fluidity. Fresh cementitious composites for extrusion purposes are dough-like, have almost no fluidity, and exhibit high cohesion and pseudoplastic behavior under normal conditions [Srinivasan et al. 1999; Zhou and Li 2005a; Li and Li 2007]. Though a number of extrusion practices have been successfully performed, little research has been carried out on the rheological behavior of extrudable fresh cementitious composites and the extrusion process itself.

Material tests and rheological measurements. The experimental setup and procedures for orifice and capillary extrusion have been presented in [Zhou 2004; Zhou and Li 2005b] while those for upsetting tests have been reported in detail in [Zhou and Li 2006]. Based on these studies, the steady-state postyield constitutive behavior of an extrudable short-fiber-reinforced fresh cementitious composite has been formulated from which the shear flow stress, $\tau$, is plotted as a function of the plastic shear strain rate, $\dot{\gamma}^{\mathrm{vp}}$. The postyield shear flow behavior of the extrudable fresh cementitious composite has been found to be satisfactorily described by the Herschel-Bulkley model [Zhou and Li 2005b] in the form

$$
\tau=\tau_{0}+k_{\mathrm{sc}}\left(\dot{\gamma}^{\mathrm{vp}}\right)^{m}
$$

where $\tau_{0}$ is the static shear yield stress, and $k_{\mathrm{sc}}$ and $m$ are the shear plastic flow consistency and the shear flow index, respectively. The values of the material parameters in (2-1) have been obtained through orifice and capillary extrusion tests as $\tau_{0}=2.15 \mathrm{kPa}, k_{\mathrm{sc}}=4.17 \mathrm{kPa} \cdot \mathrm{s}^{m}$, and $m=0.38$ [Zhou 2004; Zhou and Li 2005b]. Prior to yielding, the fresh cementitious composite exhibits elastic behavior with a constant Young's modulus of $E=8 \mathrm{kPa}$, which is obtained from its true stress versus true strain curve through upsetting tests presented in [Zhou and Li 2006]. 
Ram extrusion. The experimental setup and procedure for ram extrusion are similar to those described in [Zhou and Li 2005a], but different extrusion conditions are investigated in this research. The results of these tests are utilized to evaluate the applicability of the numerical procedure presented in this paper, combined with the elastoviscoplastic constitutive model, for describing the ram extrusion process for fresh cementitious composite.

The experimental setup for ram extrusion is shown in Figure 2, where a homemade ram extruder, with smooth-surface stainless steel barrel and die, is mounted in a servohydraulic materials test system machine. Before extrusion, a certain amount of readily prepared, highly concentrated, dough-like fresh cementitious composite is fed into the barrel, with an inner diameter of $80 \mathrm{~mm}$, of the extruder up to its brim. After this, the ram extrusion test starts, and the paste inside the barrel is driven by the ram with a constant velocity till it flows out of the die exit in a steady state.

Three sets of dies, with diameters of $8 \mathrm{~mm}, 12 \mathrm{~mm}$, and $15 \mathrm{~mm}$, are used in the experiment, resulting in extrusion ratios of $100,44.4$, and 28.4 , respectively. The die-land lengths are truncated to obtain die-land length-to-diameter ratios, $L / D$, of 0.83 and 4.79 for each set of dies. A series of tests with various extrusion velocities are conducted, representing a broad range of apparent strain rates imposed on the fresh composite. During extrusion, the data acquisition system records ram displacement, time, and extrusion load. Each test is repeated three times and the average values are taken as representative results.

A typical experimental output on the variation of extrusion load with imposed ram displacement is shown in Figure 3 on the next page. In the initial stage when the ram pushes the fresh cementitious composite against the die-land, the composite undergoes upsetting, which leads to a rapid increase in
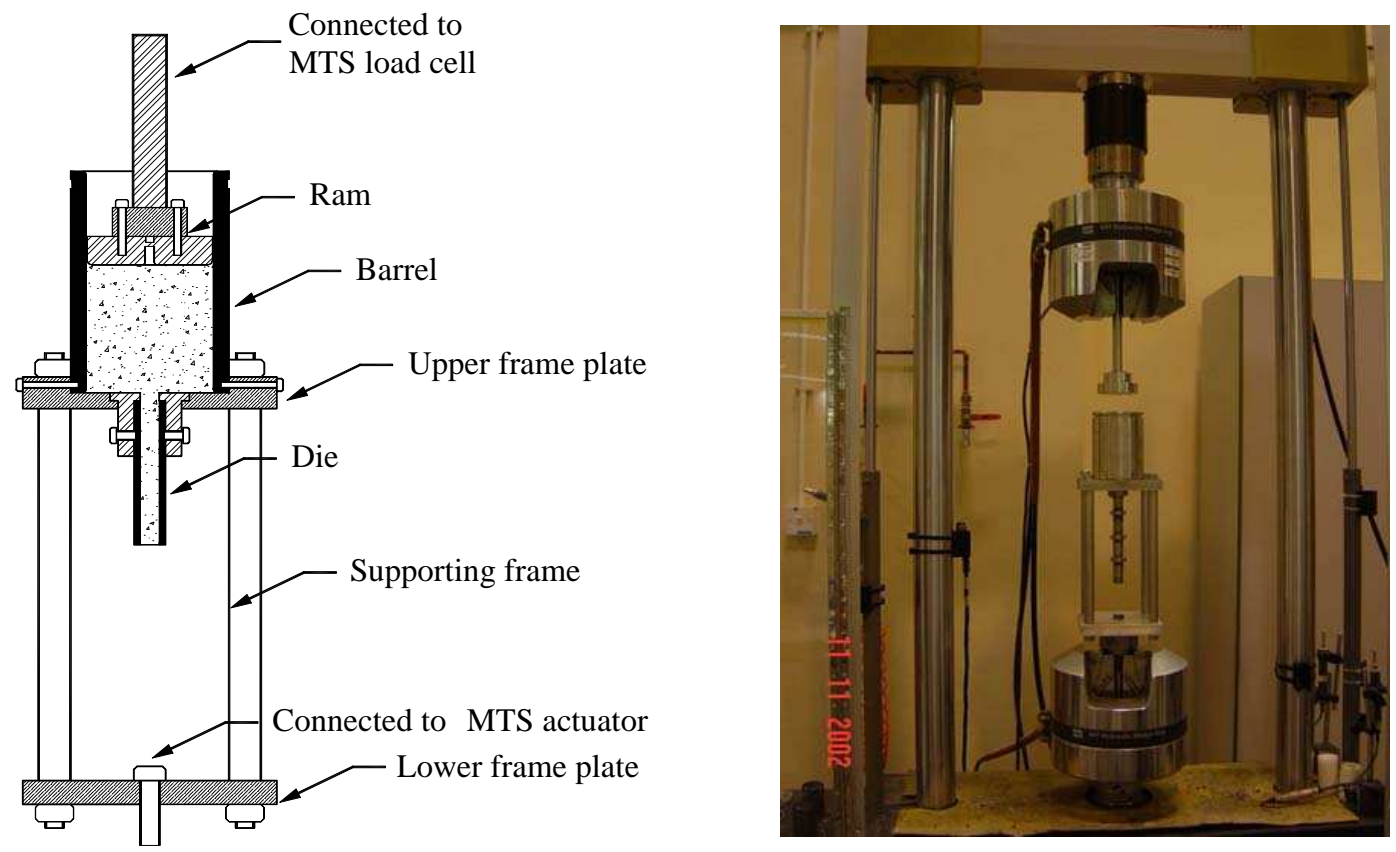

Figure 2. Experimental setup of the ram extrusion test: schematic diagram and laboratory apparatus. 


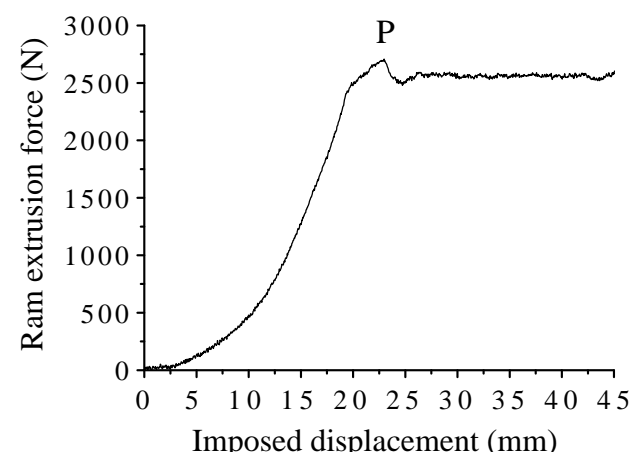

Figure 3. A typical ram extrusion load versus imposed displacement curve from the experiment.

extrusion load until it attains a peak value (point $P$ in Figure 3), where the paste breaks through the die exit. After this breakthrough, the extrusion load decreases, which is due to the reduction of the contact area, and thus friction, between the composite and the barrel. The experimental data, after the breakthrough, clearly demonstrates the attainment of a steady state extrusion (stable plateau value of the extrusion load).

\section{Numerical simulation}

Constitutive model and associated material parameters. A time-continuous model normally forms the basis for the constitutive equations in numerical simulations of elastoviscoplastic material behavior [Lof and van den Boogaard 2001]. In the time-continuous model, the relation between stress and strain, in both the elastic and the plastic domains, is usually defined in a rate formulation. Based on infinitesimal deformation theory, it is reasonable to assume that the rate of deformation can be additively decomposed into an elastic (reversible) part and an inelastic (irreversible) part, so that the total strain rate, $\dot{\varepsilon}$, can be expressed as $\dot{\varepsilon}=\dot{\varepsilon}^{\mathrm{el}}+\dot{\varepsilon}^{\mathrm{vp}}$, where $\dot{\varepsilon}^{\mathrm{el}}$ and $\dot{\varepsilon}^{\mathrm{vp}}$ represent the elastic and viscoplastic strain rates, respectively. By assuming isotropic material behavior, the elastic part is treated as linear and is expressed in Cartesian index notation as

$$
\dot{\varepsilon}_{i j}^{\mathrm{el}}=\frac{1+v}{E} \dot{S}_{i j}+\frac{1-2 v}{3 E} \dot{\sigma}_{k k} \delta_{i j}
$$

where $E$ is the Young's modulus, $v$ is the Poisson's ratio, $\dot{\sigma}$ is the rate of change of stress, and $\dot{S}$ is the rate of change of deviatoric stress. A constant Poisson's ratio, $v=0.465$, which is somewhat arbitrary, is incorporated in the constitutive model to represent nearly incompressible material behavior, which has been found to be a reasonable assumption for this highly viscous paste-like material for extrusion purposes [Zhou 2004; Zhou and Li 2006].

It has been found that the constitutive behavior of highly concentrated short-fiber-reinforced fresh cementitious paste for extrusion purposes is dominated by rheological (strain rate-dependent) effects once it deforms inelastically [Srinivasan et al. 1999; Zhou and Li 2005a; 2006; Li and Li 2007]. For simplicity, it is further assumed that the isotropic strain rate-hardening law is sufficient to describe the evolution of the flow stress of the extrudable fresh cementitious composite during plastic deformation. The strain rate-hardening law is formulated in terms of the equivalent viscoplastic strain rate, $\overline{\dot{\varepsilon}}^{\mathrm{vp}}$. Assuming an associative $J_{2}$ plastic flow where $J_{2}$ is the second invariant of the deviatoric stress tensor, the von Mises 
yield criterion is used to define the equivalent flow stress, $\bar{\sigma}$. Extending the notion of associative plasticity to viscoplasticity, the viscoplastic strain rate is assumed to be in the direction of the deviatoric stress, $S$, and is defined as

$$
\dot{\varepsilon}^{\mathrm{vp}}=\frac{3}{2} \overline{\dot{\varepsilon}}^{\mathrm{vp}} \frac{S}{\bar{\sigma}}
$$

where the equivalent viscoplastic strain rate, $\bar{\varepsilon}^{-\mathrm{vp}}$, is defined as

$$
\overline{\dot{\varepsilon}}^{\mathrm{vp}}=\sqrt{\frac{2}{3} \dot{\varepsilon}^{\mathrm{vp}}: \dot{\varepsilon}^{\mathrm{vp}}},
$$

and the equivalent flow stress, $\bar{\sigma}$, is calculated according to the von Mises yield criterion as

$$
\bar{\sigma}=\sqrt{3 J_{2}}=\sqrt{\frac{3}{2} S: S} .
$$

On the other hand, rate-dependent plasticity is often introduced by so-called overstress models, such as the Perzyna model [Perzyna 1966; 1971; Wang et al. 1997; Lof and van den Boogaard 2001; Ponthot 2002] or the Duvaut-Lions model [Simo and Hughes 1998]. Contrary to the case of rate independent plasticity, in these models the equivalent flow stress, $\bar{\sigma}$, is no longer constrained to remain less than or equal to the static yield stress, $\sigma_{0}$, but could be greater than it. The part of the stress that is outside the static yield surface, which is called the overstress, determines the viscoplastic strain rate [Ponthot 2002]. Clearly, an inelastic process can take place if, and only if, the overstress, $d=\left\langle\bar{\sigma}-\sigma_{0}\right\rangle$, is not less than zero, that is if $d \geq 0$, where $\langle x\rangle$ denotes the MacAuley brackets defined by $\langle x\rangle=1 / 2(x+|x|)$. The classical viscoplastic models of Perzyna type [1966; 1971] may be formulated as

$$
\overline{\dot{\varepsilon}}^{\mathrm{vp}}=D_{\mathrm{vp}}\left(\frac{\bar{\sigma}-\sigma_{0}}{\sigma_{0}}\right)^{1 / r},
$$

where $D_{\mathrm{vp}}$ and $r$ are the viscoplastic material parameters. This mathematical relationship describes the evolution of the equivalent viscoplastic strain rate, $\bar{\varepsilon}^{\mathrm{vp}}$, as a function of the overstress, $\bar{\sigma}-\sigma_{0}$, in the plastic domain. The material parameters in (3-5), namely $D_{\mathrm{vp}}$ and $r$, can be obtained for the extrudable fresh cementitious composite through the following procedure.

First, the shear-form Herschel-Bulkley equation, (2-1), is transformed into its pertinent uniaxial formulation following the theoretical procedure proposed in [Stouffer and Dame 1996] as

$$
\bar{\sigma}=\sigma_{0}+k\left(\overline{\dot{\varepsilon}}^{\mathrm{vp}}\right)^{n},
$$

where the material parameters $\sigma_{0}, k$, and $n$ are the uniaxial yield stress, the uniaxial plastic flow consistency, and the uniaxial plastic flow index, respectively. On the other hand, for a material that obeys the von Mises yield criterion, the uniaxial form of the Herschel-Bulkley equation may be obtained from the graph of shear stress against shear strain rate by plotting $\tau=\sigma / \sqrt{3}$ as a function of $\ldots \gamma^{\mathrm{vp}}=\sqrt{3} \dot{\varepsilon}^{\mathrm{vp}}$ from its shear-form equation [Adams et al. 1997; Aydin et al. 2000]. Substituting these relationships into (2-1) yields

$$
\sigma=\sqrt{3} \tau_{0}+(\sqrt{3})^{1+m} k_{\mathrm{sc}}\left(\dot{\varepsilon}^{\mathrm{vp}}\right)^{m} .
$$

It has been found that the uniaxial bulk flow index, $n$, in (3-6) is approximately equal to the shear flow index, $m$, in (2-1), that is, $n=m$, for the extrudable short-fiber-reinforced fresh cementitious composite investigated in this study [Zhou 2004]. In the case of uniaxial bulk flow, $\bar{\sigma}=\sigma$ and $\overline{\dot{\varepsilon}}^{\mathrm{vp}}=\dot{\varepsilon}^{\mathrm{vp}}$. Therefore, 
by comparing (3-6) and (3-7), the material parameters in (3-6) can be obtained as $\sigma_{0}=\sqrt{3} \tau_{0}$ and $k=$ $\sqrt{3}^{1+m} k_{\mathrm{sc}}$ for the extrudable fresh cementitious composite. Rewriting (3-5) in the form of (3-6) gives

$$
\bar{\sigma}=\sigma_{0}+\frac{\sigma_{0}}{D_{\mathrm{vp}}^{r}}\left(\overline{\dot{\varepsilon}}^{\mathrm{vp}}\right)^{r} .
$$

Thus, the interrelationships between the parameters in (3-6) and (3-8) are obtained as $r=m$ and $D_{\mathrm{vp}}=$ $\left(\sigma_{0} / k\right)^{1 / m}$. Therefore, the associated material parameters in (3-8) are derived as $\bar{\sigma}_{0}=3.72 \mathrm{kPa}, D_{\mathrm{vp}}=$ $0.101 \mathrm{~s}^{-1}$, and $r=0.38$, by referring to the material test results for orifice extrusion and capillary extrusion presented in [Zhou 2004; Zhou and Li 2005b].

As seen above, our elastoviscoplastic constitutive model features the strain rate-dependent von Mises yield criterion, an associative flow rule, and an nonlinear strain rate-hardening law. The rate-form constitutive model has been integrated into an incremental formulation and implemented into a numerical procedure based on the ANSYS/LS-DYNA explicit finite element code [Zhou 2004]. In this paper, various ram extrusion processes are simulated by this numerical procedure, combined with the constitutive model for the short-fiber-reinforced fresh cementitious composite for extrusion purposes.

Geometries and the finite element model. In numerical analysis, the ram and the extruder, which is composed of the barrel and the die-land, are modeled as rigid objects. Due to axisymmetry, the deformation of the paste in the radial direction at the center of the extruder is zero. Thus, only a single azimuthal slice of the ram extruder and the cementitious composite inside of the center line is modeled in the numerical analysis with a two-dimensional (2D) finite element model composed of a set of four-node isoparametric 2D solid elements, which makes remeshing easier.

Figure 4 shows a typical initial mesh, generated by the ANSYS/LS-DYNA finite element code, for the ram extrusion experimental setup investigated in this study (Figure 2). No contact element is available in explicit analysis based on the LS-DYNA finite element code; contact is achieved using a penalty

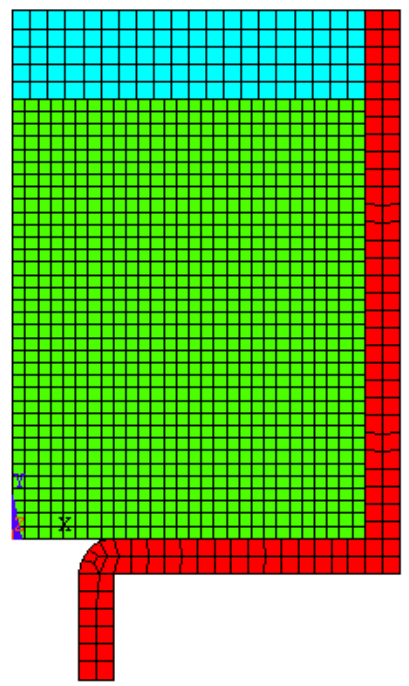

Figure 4. Initial finite element mesh for simulating the ram extrusion process with a die diameter of $15 \mathrm{~mm}$. 
approach in the LS-DYNA code, in which the fresh cementitious paste is defined as the slave surface while the walls of the extruder and the ram are assigned as the master surfaces. A Coulomb friction criterion, with a constant friction coefficient of $\mu=0.195$ obtained through upsetting tests [Zhou and Li 2006], is adopted to model boundary interaction whenever the paste contacts the walls of the extruder. A reduced kinematic value is used as the dynamic friction coefficient to simulate reduced boundary friction during dynamic loading. The fresh paste within the ram extruder is modeled by an arbitrary LagrangianEulerian (ALE) mesh as it travels through the die entry into the die-land, while the ram, the barrel, and the die-land are modeled by a much coarser Lagrangian mesh to reduce computation time. The ALE mesh is very effective for simulating large deformation and large strain problems, and allows smoothing of a distorted mesh without requiring a complete remeshing. Thus, it can largely avoid mesh distortion.

A typical initial mesh for the highly concentrated fresh cementitious paste body in the barrel contains 1200-2000 elements, the smallest element size being about $10 \%$ of the radius of the die-land. In numerical simulation, an incremental analysis is performed, in which the imposed ram displacement during each analytical step, before remeshing, is between 0.01 and $0.02 \%$ of the initial height of the fresh cementitious paste body in the barrel. A very small time step is adopted in numerical analysis to ensure stability in the fine mesh near the die entry, resulting in much longer computation time than for the simulation of the upsetting process presented in [Zhou and Li 2007]. The computation time is longer for cases with larger extrusion ratios than for those with smaller extrusion ratios. In general, remeshing is required to achieve a successful complete numerical simulation, resulting in the element number increasing during analysis.

A series of ram extrusion processes of the fresh cementitious composite, under various extrusion velocities, die-land lengths, and diameters, were simulated with the numerical procedure described above, combined with an elastoviscoplastic constitutive model proposed for the fresh cementitious composite tailored for extrusion purposes. The extrusion load can be obtained at any time during numerical analysis by integrating the longitudinal stress component, $\sigma_{y}(r)$, over the area of the ram. For an axisymmetric problem, the extrusion load, $F_{\text {ext }}$, is given by

$$
F_{\mathrm{ext}}=\int_{0}^{D_{0} / 2} 2 \pi r \sigma_{y}(r) d r
$$

where $\sigma_{y}(r)$ is the longitudinal stress of the elements contacting the ram. The extrusion load predicted at each deformation stage is then plotted against the imposed ram displacement to produce an extrusion load versus imposed displacement curve, to which the predicted extrusion load versus imposed displacement curve is referred in this paper. It is then compared with its experimental counterpart to test the applicability of the numerical procedure, combined with the constitutive model, to simulate the ram extrusion process of the fresh cementitious composite.

Comparison of numerical and experimental results. For every extrusion process, the numerical prediction is conducted from the start of extrusion until a steady state is reached. The relationship between the extrusion load and the imposed ram displacement offers a channel for comparing the numerical and experimental results quantitatively. The numerical procedure, as well as the elastoviscoplastic constitutive model for the fresh cementitious composite, presented in this study, is thus mainly verified by this comparison. Figure 5, top left, shows the predicted and experimental extrusion load versus imposed 

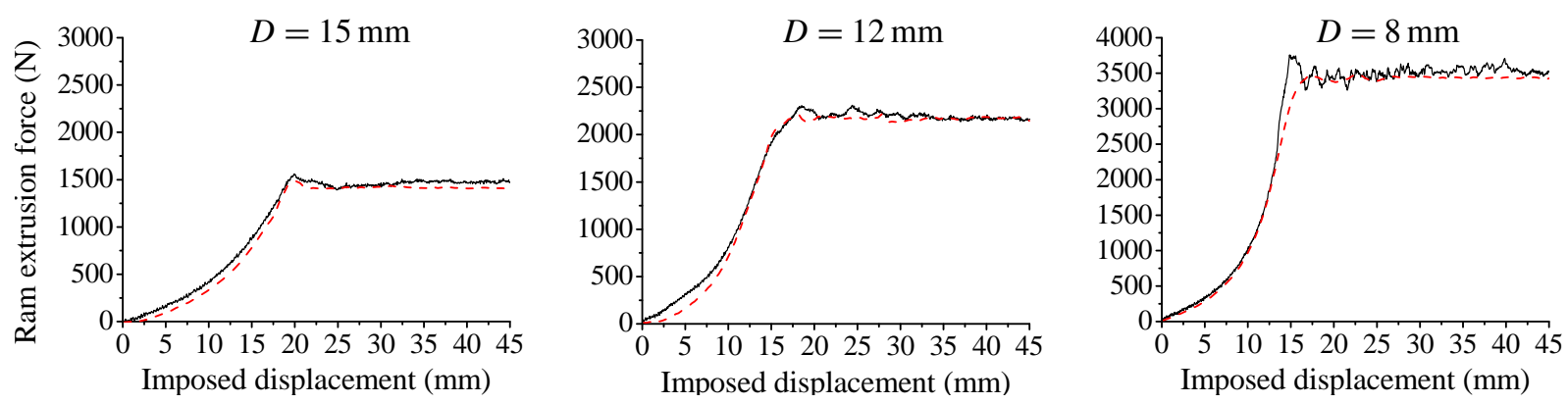

Figure 5. Curves of experimental (solid) and predicted (dashed) extrusion load versus imposed displacement, for $L / D=0.83, V=0.1 \mathrm{~mm} / \mathrm{s}$, and various values of $D$.

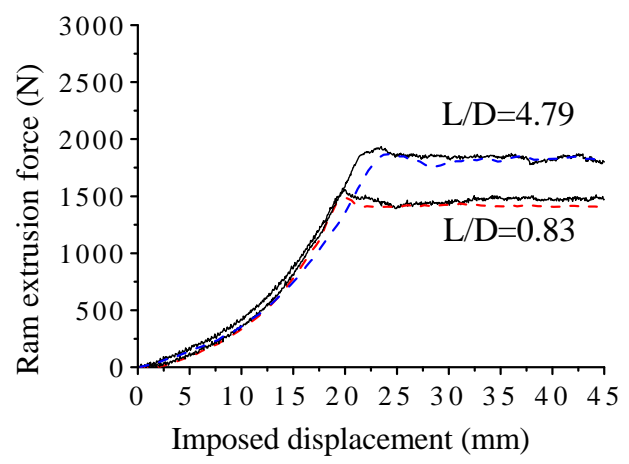

Figure 6. Curves of experimental (solid) and predicted (dashed) extrusion load versus imposed displacement, for $D=15 \mathrm{~mm}, V=0.1 \mathrm{~mm} / \mathrm{s}$, and two values of $L / D$.

displacement curves for the ram extrusion process through a $15 \mathrm{~mm}$ diameter die, with a die-land lengthto-diameter ratio of 0.83 , at an extrusion velocity of $0.1 \mathrm{~mm} / \mathrm{s}$. It can be seen that the agreement between the numerical and experimental results is good during the upsetting stage and is within 5\% difference under higher loads at steady-state extrusion. The predicted and experimental peak extrusion loads are $1485 \mathrm{~N}$ and $1562 \mathrm{~N}$, respectively. Reviewing the uncertainty in the dynamic boundary interaction, the agreement between the predicted and experimental extrusion loads should be considered satisfactory. Besides, the numerical prediction also indicates a decrease in extrusion load after the breakthrough, which is similar to that observed experimentally (as shown in Figure 3).

More results of the predicted and experimental extrusion load versus imposed ram displacement curves are shown in the rest of Figure 5 and in Figure 6 for other parameter values. The results from numerical simulation are generally seen to be in good accordance with the pertinent experimental results and the difference between the predicted and measured steady-state extrusion loads is relatively low, which further verifies that the numerical procedure and the constitutive model presented in this paper can be used with confidence for the prescribed ram extrusion conditions.

Interpretation of the paste flow in ram extrusion. Based on the verified numerical procedure, the evolution of the deformation and distribution of the state variables, such as extrusion pressure, within the paste flow are also predicted throughout the extrusion process. The paste flow in ram extrusion is thus 
interpreted based on these numerical results, which also helps in validating the numerical procedure and the constitutive model for the fresh cementitious composite qualitatively and in understanding the ram extrusion mechanism for the short-fiber-reinforced fresh cementitious composite.

Evolution of deformation. At the start of the ram extrusion process, when the ram pushes the fresh cementitious composite in the barrel, the composite paste commences upsetting. As the process continues, the paste flows towards the die-land, where the velocity is the highest within the extruder. A breakthrough extrusion load (indicated by the peak point $P$ in the extrusion load versus imposed displacement curve shown in Figure 3) is necessary for the paste to be extruded out of the die exit. Past this peak, the steadystate extrusion process starts, although the extrusion load decreases due to the drop in friction between the paste and the walls of the barrel. As seen in Figures 5 and 6, the extrusion load reaches its peak value when the paste fills the die-land and starts to emerge, and then it decreases.

The deformation $U_{y}$ of the composite along the extrusion direction is shown in Figure 7, for a ram extrusion process with a $30 \mathrm{~mm}$ diameter die under a ram velocity of $0.1 \mathrm{~mm} / \mathrm{s}$, at ram imposed displacements of $1 \mathrm{~mm}$ and $4.2 \mathrm{~mm}$. It can be seen that the deformation of the composite in the die-land is significantly higher than that in the barrel, because of the high extrusion ratio. In the corner between the die-land and the barrel, which is referred as the static zone (Figure 7), the deformation of the composite is close to zero. The outline of the static zone, however, is not so obvious. When the composite travels in the die-land, a nonuniform displacement field occurs. It can be seen that the composite near the walls of the barrel and the die-land flows more slowly than the paste in the inner region, due to the boundary friction existing between the composite paste and the walls of the extruder.
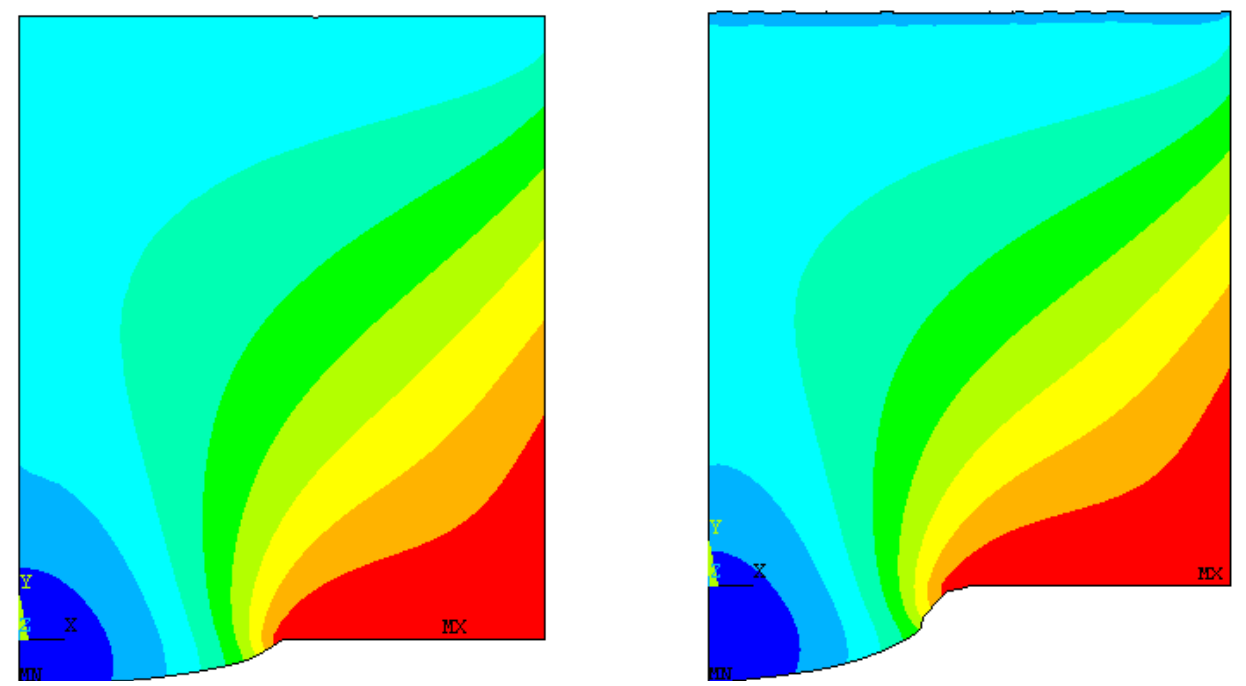

Figure 7. Deformation $U_{y}$ (average) of the fresh paste flow along the extrusion direction, at an imposed ram displacement of $1 \mathrm{~mm}$ (left) and $4.2 \mathrm{~mm}$ (right). Color bands range in equal increments from the minimum value, represented by blue and achieved at the bottom left of each diagram, to the maximum, represented by red at the bottom right. The intervals are $[-1.290,-0.001]$ for the left diagram and $[-5.292,0.017]$ for the right diagram (in $\mathrm{mm}$ ). 

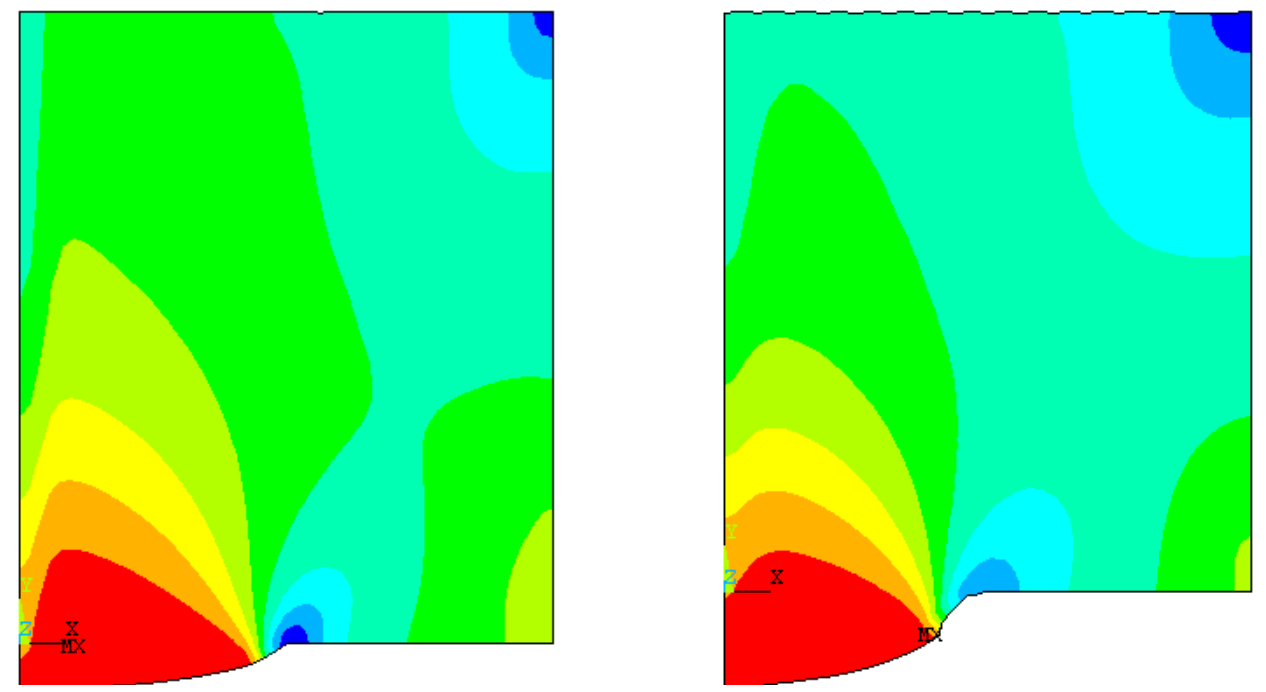

Figure 8. Extrusion pressure $S_{y}$ (average) within the paste flow along the extrusion direction, at an imposed ram displacement of $1 \mathrm{~mm}$ (left) and $4.2 \mathrm{~mm}$ (right). Color bands range in equal increments from the minimum value, represented by blue and achieved at the top right (and nearly so at the concave corner) of each diagram, to the maximum, represented by red at the bottom left. The intervals are $[-915,5]$ for the left diagram and $[-3670,43]$ for the right diagram (in $\mathrm{kPa}$ ).

Distribution of state variables within the paste flow. The distribution of the extrusion pressure $S_{y}$ along the extrusion direction is shown in Figure 8 at imposed ram displacements of $1 \mathrm{~mm}$ and $4.2 \mathrm{~mm}$ for a ram extrusion process with a $30 \mathrm{~mm}$ diameter die under a ram velocity of $0.1 \mathrm{~mm} / \mathrm{s}$. In general, negative stress is found from these figures, which is representative of an essential compression-dominant state. However, tensile stress exists in the front surface of the paste flow, owing to the surface friction at the die-land wall, which restricts the paste flow. As a result, the front surface of the paste flow is mainly subjected to tension (see Figure 8). Tensile stress in the front surface of the paste flow is mainly due to two factors: first, the material in the inner region flows faster than that near the die-land wall; and second, the friction between the composite paste and the die-land wall restricts the paste flow. It could also be ascribed to gravity as well.

Effects of extrusion ratio and extrusion velocity. The effect of the extrusion ratio on the extrusion load is demonstrated in Figure 9 for extrusion processes with a $12 \mathrm{~mm}$ diameter die and a $15 \mathrm{~mm}$ diameter die at a ram velocity of $0.2 \mathrm{~mm} / \mathrm{s}$. An increase in the extrusion ratio, that is, a decrease in die diameter, corresponds to an increase in the apparent strain rate. Since the fresh cementitious composite is a strain rate-hardening material, its flow stress increases with the strain rate. Thus more work is required to extrude the composite out of the die-land with a smaller diameter than that with a larger diameter, resulting in an increase in extrusion load. It can also be seen from Figure 9 that the extrusion load increases more rapidly till reaching the steady-state value for the extrusion process with a higher extrusion ratio.

The effect of the extrusion velocity on the extrusion load can be seen in Figure 10, where the numerical and experimental results are shown for the extrusion processes at velocities of $0.1 \mathrm{~mm} / \mathrm{s}$ and $0.2 \mathrm{~mm} / \mathrm{s}$ 


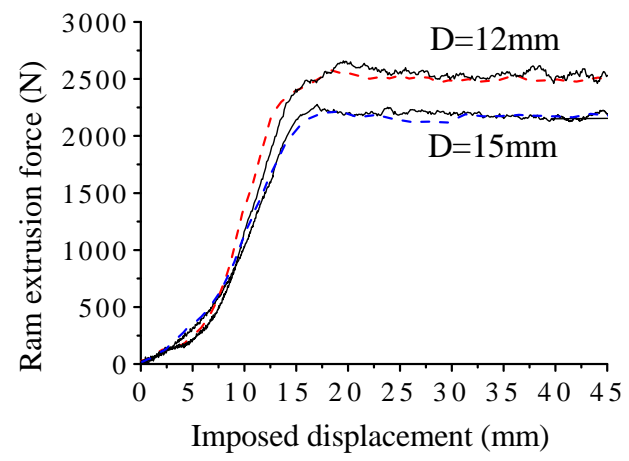

Figure 9. Curves of experimental (solid) and predicted (dashed) extrusion load versus imposed displacement $(L / D=0.83$ and $V=0.2 \mathrm{~mm} / \mathrm{s})$.

with a die diameter of $12 \mathrm{~mm}$ and a die-land length-to-diameter ratio of 0.83 . Again, since the fresh cementitious composite is a strain rate-hardening material, its flow stress increases as its strain rate increases. An increase of extrusion velocity results in an increase of apparent strain rate, and thus equivalent flow stress and, consequently, extrusion load as can be seen from Figure 10 in which the extrusion load for the process under the extrusion velocity $0.2 \mathrm{~mm} / \mathrm{s}$ is much higher than under the extrusion velocity $0.1 \mathrm{~mm} / \mathrm{s}$. Again, the extrusion load in the former extrusion process increases more rapidly to a steady-state value than for the latter.

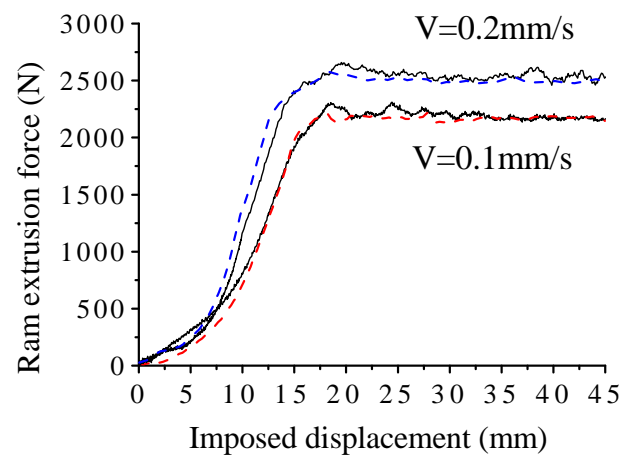

Figure 10. Curves of experimental (solid) and predicted (dashed) extrusion load versus imposed displacement $(L / D=0.83$ and $L=12 \mathrm{~mm})$.

\section{Conclusions}

A numerical procedure based on the explicit ANSYS/LS-DYNA finite element code, combined with an elastoviscoplastic constitutive model for the extrudable fresh cementitious composite, has been established to simulate a series of ram extrusion processes. The predictions of extrusion load versus imposed displacement are compared with experimental results. The evolution of deformation and the distribution of state variables within the composite paste flow in the ram extrusion process are then interpreted based on numerical results and the ram extrusion mechanism. The effects of the extrusion ratio and extrusion 
velocity on extrusion load are discussed. Based on experimental and numerical investigations, the main conclusions that can be drawn are the following:

- The extrusion load versus imposed displacement predictions agree well with the experimental results for various extrusion processes, suggesting that the numerical procedure established and the constitutive model proposed for the fresh cementitious composite can be used with confidence for the prescribed ram extrusion processes. The extrusion load increases more rapidly to a steady-state value in cases of greater extrusion ratio and/or higher ram velocity. In these processes, the steadystate extrusion loads are also greater.

- The evolution of deformation, as well as the distribution of state variables within the paste flow, predicted by numerical analyses, gives a promising interpretation of the fresh cementitious composite paste flow in various ram extrusion processes. Due to surface friction existing between the paste flow and the walls of the extruder, the paste in the central region moves faster than that along the walls of the extruder, resulting in the composite at the front surface being mainly under tension, while the composite in other regions is mainly subjected to compression.

- The numerical procedure presented in this paper shows the potential for improving the understanding of the flow behavior of paste-like materials in extrusion, which might be applied to forming processes for other concentrated paste-like semisolid elastoviscoplastic materials.

\section{References}

[Adams et al. 1997] M. J. Adams, I. Aydin, B. J. Briscoe, and S. K. Sinha, "A finite element analysis of the squeeze flow of an elasto-viscoplastic paste material”, J. Non-Newton. Fluid Mech. 71:1-2 (1997), 41-57.

[Aldea et al. 1998] C. Aldea, S. Marikunte, and S. P. Shah, "Extruded fiber reinforced cement pressure pipes", Adv. Cem. Based Mater. 8:2 (1998), 47-55.

[Antúnez 2000] H. J. Antúnez, "Linear elements for metal-forming problems within the flow approach", Comput. Methods Appl. Mech. Eng. 190:5-7 (2000), 783-801.

[Aydin et al. 2000] I. Aydin, F. R. Biglari, B. J. Briscoe, C. J. Lawrence, and M. J. Adams, "Physical and numerical modelling of ram extrusion of paste materials: conical die entry case", Comput. Mater. Sci. 18:2 (2000), 141-155.

[Benbow and Bridgwater 1993] J. J. Benbow and J. Bridgwater, Paste flow and extrusion, Clarendon, Oxford, 1993.

[Benbow et al. 1991] J. J. Benbow, S. H. Jazayeri, and J. Bridgwater, "The flow of pastes through dies of complicated geometry", Powder Technol. 65:1-3 (1991), 393-401.

[Blackburn and Böhm 1994] S. Blackburn and H. Böhm, "The influence of powder packing on the rheology of fibre-loaded pastes", J. Mater. Sci. 29:16 (1994), 4157-4166.

[Chou et al. 2003] S. Chou, K. Sydow, P. J. Martin, J. Bridgwater, and D. I. Wilson, "Stress relaxation in the extrusion of pastes", J. Eur. Ceram. Soc. 23:5 (2003), 637-646.

[Li and Li 2007] Z. Li and X. Li, "Squeeze flow of viscoplastic cement-based extrudate", J. Eng. Mech. (ASCE) 133:9 (2007), 1003-1008.

[Li and Mu 1998] Z. Li and B. Mu, "Application of extrusion for manufacture of short fiber reinforced cementitious composite", J. Mater. Civ. Eng. (ASCE) 10:1 (1998), 2-4.

[Li et al. 1999] Z. Li, B. Mu, and S. N. C. Chui, "Systematic study of properties of extrudates with incorporated metakaolin or silica fume", ACI Mater. J. 96:5 (1999), 574-580.

[Li et al. 2001] Z. Li, B. Mu, and S. N. C. Chui, "Static and dynamic behavior of extruded sheets with short fibers", J. Mater. Civ. Eng. (ASCE) 13:4 (2001), 248-254. 
[Li et al. 2004] Z. Li, X. Zhou, and B. Shen, "Fiber-cement extrudates with perlite subjected to high temperatures", J. Mater. Civ. Eng. (ASCE) 16:3 (2004), 221-229.

[Lof and van den Boogaard 2001] J. Lof and A. H. van den Boogaard, "Adaptive return mapping algorithms for $J_{2}$ elastoviscoplastic flow”, Int. J. Numer. Methods Eng. 51:11 (2001), 1283-1298.

[Peled and Shah 2003] A. Peled and S. P. Shah, "Processing effects in cementitious composites: extrusion and casting", $J$. Mater. Civ. Eng. (ASCE) 15:2 (2003), 192-199.

[Perzyna 1966] P. Perzyna, "Fundamental problems in viscoplasticity", pp. 243-377 in Advances in applied mechanics, vol. 9 , edited by G. Kuerti, Academic Press, New York, 1966.

[Perzyna 1971] P. Perzyna, "Thermodynamic theory of plasticity", pp. 313-355 in Advances in applied mechanics, vol. 11, edited by C.-S. Yih, Academic Press, New York, 1971.

[Ponthot 2002] J. P. Ponthot, "Unified stress update algorithms for the numerical simulation of large deformation elasto-plastic and elasto-viscoplastic processes", Int. J. Plast. 18:1 (2002), 91-126.

[Shao and Shah 1997] Y. Shao and S. P. Shah, "Mechanical properties of PVA fiber reinforced cement composites fabricated by extrusion processing", ACI Mater. J. 94:6 (1997), 555-564.

[Shao et al. 1995] Y. Shao, S. Marikunte, and S. P. Shah, "Extruded fiber-reinforced composites", Concr. Int. 17:4 (1995), $48-52$.

[Simo and Hughes 1998] J. C. Simo and T. J. R. Hughes, Computational inelasticity, Interdisciplinary Applied Mathematics 7, Springer, New York, 1998.

[Srinivasan et al. 1999] R. Srinivasan, D. DeFord, and S. P. Shah, "The use of extrusion rheometry in the development of extruded fiber-reinforced cement composites", Concr. Sci. Eng. 1:1 (1999), 26-36.

[Stouffer and Dame 1996] D. C. Stouffer and L. T. Dame, Inelastic deformation of metals: models, mechanical properties and metallurgy, Wiley, New York, 1996.

[Wang et al. 1997] W. M. Wang, L. J. Sluys, and R. de Borst, "Viscoplasticity for instabilities due to strain softening and strain-rate softening", Int. J. Numer. Methods Eng. 40:20 (1997), 3839-3864.

[Zhou 2004] X. Zhou, Rheological behaviors of the fresh SFRCC extrudate: experimental, theoretical and numerical investigations, Ph.D. thesis, Hong Kong University of Science and Technology, Hong Kong, 2004.

[Zhou and Li 2005a] X. Zhou and Z. Li, "Characterization of rheology of fresh fiber reinforced cementitious composites through ram extrusion", Mater. Struct. 38:275 (2005), 17-24.

[Zhou and Li 2005b] X. Zhou and Z. Li, "Characterizing rheology of fresh short fiber reinforced cementitious composite through capillary extrusion”, J. Mater. Civ. Eng. (ASCE) 17:1 (2005), 28-35.

[Zhou and Li 2006] X. Zhou and Z. Li, "Upsetting tests of fresh cementitious composites for extrusion", J. Eng. Mech. (ASCE) 132:2 (2006), 149-157.

[Zhou and Li 2007] X. Zhou and Z. Li, "Numerical simulations of upsetting process of the fresh fiber-cement paste", J. Eng. Mech. (ASCE) 133:11 (2007), 1192-1199.

Received 11 Feb 2009. Revised 30 Jun 2009. Accepted 4 Jul 2009.

XIANgming ZhOU: Xiangming. Zhou@brunel.ac.uk

Brunel University, School of Engineering and Design, Kingston Lane, Uxbridge UB8 3PH, Middlesex, United Kingdom

ZONGJIN LI: zongjin@ust.hk

Hong Kong University of Science and Technology, Department of Civil and Environmental Engineering, Clear Water Bay,

Kowloon, Hong Kong 